\begin{tabular}{|c|c|c|}
\hline Beitr. Ent. & Keltern & ISSN 0005-805X \\
\hline $\mathbf{5 6}(2006) 2$ & S. 334-346 & 15.12 .2006 \\
\hline
\end{tabular}

\title{
Die Käfer der Paläarktis: Probleme und Perspektiven
}

\author{
Mit 2 Figuren
}

IVAN LÖBL

\section{Zusammenfassung}

Taxonomie liefert grundlegende Kenntnisse über die Vielfalt des Lebens sowie eine universelle Sprache, die eine eindeutige Übertragung biologischer Informationen ermöglicht. Trotzdem ist ein Rückgang der Taxonomie in vielen europäischen Ländern und eine fortschreitende Krise der Bio-Information zu registrieren. Erfahrungen mit dem Katalog der paläarktischen Käfer und der Kontakt zu zahlreichen Koleopterologen zeigen, dass die gegenwärtige Behinderung der Taxonomie ein Resultat mehrere Faktoren ist. Als entscheidend dürften die Unterschätzung der Variabilität der Lebewesen, der Glaube an neue Technologien, die der taxonomischen Realität nicht adaptierte Wertung wissenschaftlicher Arbeit, die Konfusion von Taxonomie mit ihrer Bestimmungsfunktion und die Erwartung endgültiger Erkenntnisse sein. Methoden, die eine Überwindung der Probleme versprechen, sind diskutiert worden. Entweder fußen sie auf fraglichen Konzepten oder sie könnten zwar die gegenwärtige Lage verbessern, jedoch ohne eine nötige Wende zu bewirken. Es scheint, dass ein wirklicher Fortschritt nur durch angemessene Unterstützung und eine von $a$ priori befreite Wertung der Taxonomie (und Systematik) in den Universitäten und Museen erreicht werden kann. Sollten die gegenwärtigen Tendenzen andauern, wird man die professionelle Taxonomie und die mit ihr zusammenhängenden Kenntnisse und Erkenntnisse in viele europäische Länder importieren müssen.

\section{Summary}

Taxonomy provides basic information about the diversity of life, and a universal language that unambiguously vehicles such information. Nevertheless, a demise of taxonomy is widespread in many European countries and results in a bio-information crisis. Based on experience with Palaearctic Coleoptera and a large number of coleopterists, the taxonomic impediment is seen as a consequence of a number of factors. Among them, the underestimation of the variability of species, the believe in new technologies that are assumed to provide more relevant data than complex knowledge, the inadequate means of evaluation of work in taxonomy, the confusion of taxonomy with its means of identification, and the expectation of finality in taxonomic research appear to have a particularly negative impact. New methods proposed as salvage for taxonomy are discussed. They appear either ill-based, or may punctually approve deficits, but luck potential to reverse the present situation. It is suggested that true progress requires adequate support, and a priori free re-evaluation of taxonomy (and biosystematics) in universities as in museums. Should the present tendencies in many European countries last, the professional taxonomy and associated knowledge will have to be imported, or may vanish.

\section{Keywords}

Bio-information crisis, taxonomy, impediments, perspectives. 


\section{Einleitung}

Käfer stellen bekanntlich die artenreichste Ordnung im Tierreich. Schätzungsweise wurden bisher 350000 bis 400000 Käferarten beschrieben. Die etwa 10 prozentige Spanne ist symptomatisch für den Kenntnisstand. Sie ist auf Lücken in den Daten-Quellen zurückzuführen: auf Kataloge, die entweder veraltet oder inhaltsmäßig beschränkt sind, und auf den Zoological Record, der nicht alle neuen Taxa, vor allem nicht alle taxonomischen und nomenklatorischen Änderungen registriert.

Kataloge liefern eine Übersicht über die Vielfalt einer Gruppe. Im besten Falle enthalten sie alle publizierten taxonomischen und nomenklatorischen Aktionen, ausreichende Informationen über die Verbreitung der Arten und Unterarten sowie Hinweise auf andere wichtige Daten, wie z. B. über den Verbleib des Typus-Materials, über Referenzen zu den Entwicklungsstadien, die Lebensweise und auch auf praktikable Bestimmungstabellen. Solche modernen „vollständigen“ Kataloge sind A Catalogue of Coleoptera of America North of Mexico (1978-1997, United States Department of Agriculture, Washington DC, USA), World Catalogue of Insects (1998- , Apollo Books, Stenstrup, Denmark) oder der Zoological Catalogue of Australia (1983- , Australian Government Publishing Service, Canberra, Australia). Immerhin hat der amerikanische Katalog seit Erscheinen des ersten Bandes im Jahre 1978 nur einen Bruchteil der nearktischen Fauna behandelt und ist nicht weiter geführt worden. Der zweite Katalog besteht seit 7 Jahren und enthält in vier Bänden zwar die meisten Wasserkäfer, doch werden damit nur etwa $5 \%$ der gesamten Käferfauna erfasst. Um die dritte Katalogserie steht es nicht viel besser, obwohl die australische Fauna relativ überschaubar zu sein scheint.

Die allgemein bekannten Kataloge über die Käfer der Paläarktis, die in der Praxis als Grundlage für faunistische und andere Studien dienen, sind alle veraltet. Darüber hinaus enthalten sie unzählige Fehler, die immer wieder neu abgeschrieben wurden. Gegenwärtig besitzt niemand einen korrekten Gesamtüberblick über die Käferfauna der Paläarktis. Es ist ein Paradoxon, dass die heute allgemein zugänglichen Dateien über neuweltliche Käfer unsere Kenntnisse genauer wiedergeben als jene über die „Paläarktis“, trotz der doch zahlreicheren altweltlichen Taxonomen.

Dass formelle Fehler etwa zwei Jahrhunderte lang bestehen können, sollen folgende drei Beispiele, aus Legionen ähnlicher Fälle ausgewählt, zeigen:

a. ohne Einfluss auf die Nomenklatur: Tenebrio gigas Linnaeus, 1767: 674 aus Amerika und Tenebrio gigas Linnaeus, 1767: 676 aus dem mediterranen Gebiet wurden gleichzeitig beschrieben. Linnaeus hat den zweiten Namen in den "Errata" seines Werkes durch den Namen Tenebrio gages ersetzt. Daher sollte die weit verbreitete und gut bekannte Art nicht Blaps gigas Linnaeus, 1767, sondern Blaps gages heißen. Die Nomenklatur erlaubt es, den unrichtigen, aber allgemein gebrauchten Namen zu behalten.

b. mit geringem Einfluss auf die Nomenklatur: die Scarabaeiden-Gattung Oniticellus ist zum erstenmal gültig von Dejean (1821), und nicht von Serville eingeführt worden.

c. mit größerem Einfluss auf die Nomenklatur: Isocerus LATREILle, 1802 ist ein Synonym bei den Cerambycidae, während bei den Tenebrionidae als valid sein jüngeres Hononym Isocerus DejEAN, 1821 gebraucht wird und muss deshalb durch einen neuen Namen ersetzt werden.

Ein neu kompilierter Katalog der Käfer der Paläarktis, der unter anderen auch solche Fehler beseitigt, ist seit langem fällig. Aus Erfahrungen mit gescheiterten, sehr langsam realisierten oder zu oberflächlich kompilierten Katalogen und Checklisten haben die 
Herausgeber ihre Anforderungen an den neuen "Catalogue of Palaearctic Coleoptera“ (siehe Löвl \& SMetana 2003, 2004) auf folgende Fragen beschränkt:

- Welche Taxa sind aus der Paläarktis bekannt geworden?

- Kann man die grobe Verbreitung der Taxa in einer brauchbaren Form vorlegen, so dass sie als Grundlage für weitere Studien nutzbar wird?

- Können korrekte Angaben (besonders die richtige Orthographie der Namen der Taxa und der Autoren) eingeführt werden und damit zu einer besseren Stabilität der Nomenklatur beitragen?

- Kann man überhaupt noch ein größeres, internationales Projekt unabhängig von öffentlichen Planungs-, Finanz- und administrativen Strukturen verwirklichen?

Während die erste und zweite Frage die biologische Realität ansprechen, hängt die dritte Frage mit dem Bedürfnis effizienter Informationsübermittlung zusammen: Da ein Binomen allein als eindeutiger Träger einer Information nicht ausreicht, werden zusätzliche Angaben, und zwar der Name des Autors, das Jahr und oft auch die Seite der ersten Publikation gebraucht. Gegenwärtig findet man häufig verschiedene Schreibweisen für einen und denselben Autor (z. B. Jacobsohn-Jacobson-Jakobson) und die verzeichneten Publikationsdaten weichen oft ab (z. B. bei Dejeans Katalog, Motschulskys Etudes entomologiques und den Jahrgängen vieler Zeitschriften). Weiterhin ist die korrekte Festlegung der Typus-Arten der Gattungen wichtig. Dies wurde bisher weitgehend vernachlässigt obwohl es eine erhöhte Instabilität der Gattungsnamen zur Folge haben kann.

Eine korrekte Datei ist im Zeitalter der Elektronik besonders notwendig geworden: die kleinste Abweichung in der Schreibweise kann zum Verlust einer Information führen. Sie verlangt aber eine Überprüfung der primären Literaturquellen, grob geschätzt etwa 40.000 Referenzen über paläarktische Käfer.

\section{Probleme}

Es zeigte sich, dass die ungenügende oder fehlende taxonomische Expertise bei zahlreichen Gruppen eines der am schwierigsten überwindbaren Hindernisse zu sein scheint, besonders dort, wo nicht nur rein kompilatorische Arbeit verlangt wurde. Darüber hinaus kamen psychologisch bedingte Schwierigkeiten hinzu. Manche Autoren wollen die bestehenden taxonomischen Probleme lösen, ohne Rücksicht auf bestehende zeitliche Limits; andere dagegen begnügen sich mit einer oberflächlich verfassten Datei, auch durch bewusstes Ignorieren der Anforderungen.

Als unerwartet erwies sich dagegen die weit verbreitete Unklarheit über die Grundlagen der Phylogenie, Systematik und Klassifikation. Das Fehlen von theoretischen Grundlagen scheint dabei eher eine Spezialität der altweltlichen Taxonomen zu sein: auf der anderen Seite des Ozeans werden Texte wie Wiley's (1981) „Phylogenetics“, gelesen.

Die Klassifikation, einmal vorgeschlagen „von oben nach unten“ (Fig. 1), das andere Mal „von unten nach oben“ (Fig. 2)und das Fehlen eines Konsensus sind für die europäische Taxonomie und Systematik symptomatisch. Als Beispiele sollen hier zwei parallele Systeme innerhalb der Staphylinoidea gezeigt werden:

In beiden Fällen sind nur Monophyla (im Sinne von Hennig 1966 oder Farris 1974) verwendet worden. Trotzdem sind die Ergebnisse recht unterschiedlich. Aus zweitem Fall resultiert eine größere Zahl taxonomischer Namen, eine Minderung der Verständlichkeit der Sprache und eine Asymmetrie in der Klassifikation der höheren Taxa. 


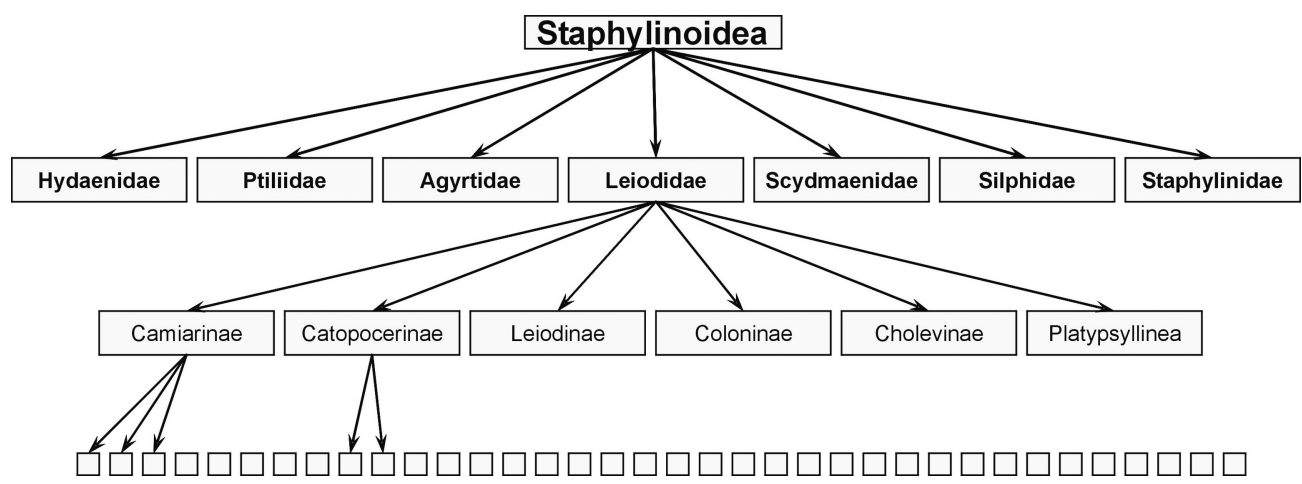

Fig. 1: Klassifikation der Staphylinoidea „von oben nach unten“.

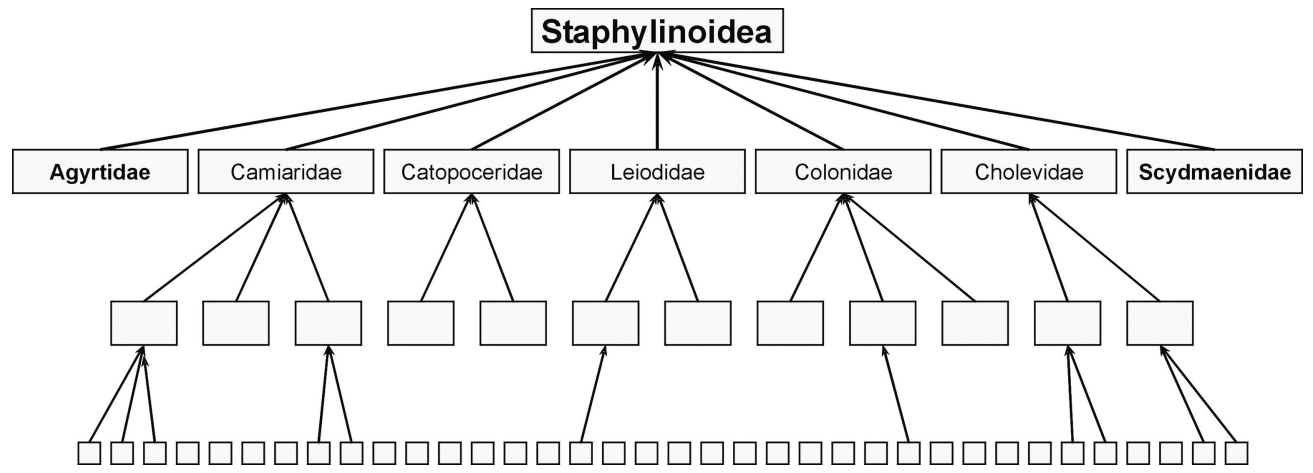

Fig. 2: Klassifikation der Staphylinoidea „von unten nach oben“.

Wenn es um Verschiebungen im Rang der Namen der Gattungs-Gruppe geht, folgt noch eine erhöhte Gefahr der Produktion sekundärer Homonyme.

Ähnlich die Scarabaeiden: sie werden als eine einzige Familie angesehen oder auf zahlreiche Familien verteilt, Bembidion- oder Pterostichus-Arten sind in eine oder in viele Gattungen gruppiert etc. Die Aporie widersprüchlicher Klassifikation ist gegenwärtig bei den Aphodiini realisiert.

Zusätzlich mussten wir feststellen, dass manche Taxonomen, unabhängig ob professionelle oder Amateure, mit oder ohne Universitätsausbildung, die Nomenklaturregeln ungenügend kennen, nicht klar verfügbare von nicht verfügbaren taxonomischen Namen unterscheiden und gewisse Bestimmungen des ICZN unrichtig interpretieren (insbesondere die sieben Zeilen des Artikels 45.6.4 über infra-subspezifische Namen und des Artikels 23.9.5 über primäre Homonyme). Dies hat einen beträchtlichen Einfluss im Bereich der Sprache. Beispielweise ist eine weit verbreitete und seit etwa 200 Jahren gut bekannte Art der Gattung Geotrupes entweder als spinipes Marsham oder als puncticollis MaLINOwsKy benannt, je nach Applikation der nomenklatorischen Regeln. Dazu kommt, dass Namen der Taxa oft mit den Taxa verwechselt worden sind (also eine Konfusion der Form mit dem Inhalt) was sich in einer undurchsichtigen Synonymie widerspiegelt.

Es zeigte sich auch, dass die Unterlagen der meisten aktiven Taxonomen den Ansprüchen des neuen Katalogs nicht genügten. Für viele ist der Zugang zu den nötigen Literaturquellen sehr schwierig. Diese Hindernisse konnten erst nach und nach durch vielseitige Unterstützung, besonders des Deutschen Entomologischen Instituts in 
Müncheberg, des Zoologischen Museums in Berlin, des American Museum of Natural History in New York und des Naturhistorischen Museums in Wien überwunden werden. Sie rauben aber in jedem Falle außerordentlich viel Zeit und Energie.

\section{Die geographischen Information}

Die Grenzen der Paläarktis sind zum Teil willkürlich bestimmt und geographische Kriterien haben sich als nicht anwendbar für unsere Arbeit erwiesen. Zum Beispiel: der „paläarktische“ oder „orientalische“ Charakter des Himalaja und der Berge Südchinas wird nicht nur durch die Höhe, sondern auch durch die Exposition beeinflusst. Es gibt auch zahlreiche, nicht wieder gefundene Arten, die aus „Arabien“ oder aus „China“ beschrieben worden sind. Und wir wissen nicht, ob sie nun in Aden oder Jordanien, in Hainan oder Xinjiang leben. Deswegen ist eine erweiterte Paläarktis in Betracht gezogen worden.

Die Kenntnisse über die Verbreitung derTaxa fußen, unter anderem, auf der Verlässlichkeit der Bestimmungen. Jedoch: ein Grossteil der bestimmten Belege wird in musealen Sammlungen aufbewahrt. Diese enthalten aber zu einem hohen Prozentsatz unrichtig bestimmte Arten. Früher hat man die Fehlbestimmungen in zahllosen faunistischen Beiträgen publiziert, heute werden sie eher in Datenbanken aufgenommen.

Hier begegnet man einem weiteren Paradoxon: die mühsam zusammengestellten Datenbanken der Käfer verschiedener Länder, Gebiete und Sammlungen sind von vielen Koautoren des Kataloges nicht berücksichtigt worden. Taxonomen geben meist ihren eigenen Erfahrungen und Kenntnissen den klaren Vorrang und kombinieren das mit den für verlässlich gehaltenen, publizierten Quellen.

Eine Darstellung der Verbreitung nach Ländern, mit einer Unterteilung von Russland in sechs Gebiete und China in Provinzen, und mit einer zusätzlichen Information über die Areale der Endemiten, schien uns der einzig praktikable Weg zu sein. Und dass die so vorgelegte Datei auch theoretisch nutzbar sein kann, zeigten aleatorische Tests mit den „chorologischen Kategorien“. Als Beispiel zwei Artenpaare:

\section{Als „Zentralasiatisch-europäisch“ angesehene Arten:}

Rybaxis longicornis LEACH, 1817: 85 (Bryaxis) E: AB AR AU BE BH BU BY CR CT CZ DE EN FI FR GB GE GR HU IR IT LA LT MC NL NT PL PT RO SK SL SP ST SV SZ UK YU N: AG MO TU A: AF ES IN IQ TD TM TR ZU - kommt auch in Nordafrika und Nordeuropa vor.

Ctenistes palpalis ReICHENBACH, 1816: 76 E: AB AL AR AU BU CR CT CZ FR GE GG GR HU IT PL PT RO SK SL SP ST SZ TR UK YU A: AF CY IN IS JO KI LE SY TM TR UZ ORR - fehlt in Nordafrika, Nordeuropa, kommt aber auch in Israel, Jordanien, Libanon und Syrien vor und transgrediert in die Orientalis.

\section{Für „Europäisch“ gehaltene Arten:}

Euplectus signatus ReIchenbach, 1816: 73 (Pselaphus) E: AU BE BH BU CR CT CZ DE EN FI FR GB GE GR HU IT LA LS LT MC NL NR NT PL RO SK SL SP ST SV SZ UK YU „Caucasus“ A: ES FE IS KI - also eine in der Realität weit, von Westeuropa bis nach dem Fernen Osten, verbreitete Art.

Tychobythinus glabratus RyE, 1870: 33 (Bythinus) E: BE CR FR GB GE IT SL SZ - beschänkt auf den westlichen Teil Europas und fehlt im Norden. 
Perspektiven für den Katalog

Etwa 60000 verfügbare taxonomische Namen mit 15000 primären Referenzen sind im 1. und 2. Band schon erfasst. Die Arbeit am 3. und 4. Band wird bald abgeschlossen sein. Es scheint möglich, beide im Jahr 2006 zu publizieren. Weitere 4 Bände dürften in nicht allzu langen Abständen erscheinen. Noch kennen wir nicht die Zahl der insgesamt aus der Paläarktis bekannt gewordenen Käferarten, sie dürfte aber 100000 deutlich übersteigen. Das Verhältnis zwischen Publikationen und gültigen Taxa ist etwa $1 \mathrm{zu} 2$ : anscheinend sind zu viele kurze Studien publiziert worden. Neue Taxa werden laufend aus den mediterranen, zentralasiatischen und ostasiatischen Ländern beschrieben. Die Schwerpunkte der neu entdeckten Taxa verschieben sich allerdings mit der Zeit und zeigen deutliche politische Korrelationen: gegenwärtig scheinen sie sich vom Himalaja in Richtung China zu verlagern.

Perspektiven für die Taxonomie der Käfer und die Taxonomie im Allgemeinen

Es gibt kaum entomologische Versammlungen, ohne dass dabei über die gegenwärtige Lage der Taxonomie und der Systematik diskutiert wird. Man spricht über eine „bioinformation crisis“. Schon 1976 berichtete R. TAYLOR über ein „taxonomic impediment“ (eine Behinderung der Taxonomie), seitdem ist dieser Termin zum Begleitbegriff der Taxonomie geworden. Ein drastischer Rückgang von Taxonomen, ganz besonders der professionellen, wird weltweit registriert. Auf diese Lage haben auch schon WHeELER (1995) und Disney (1999) hingewiesen. In Frankreich wird „l'extinction des taxonomistes" konstatiert. Gegenwärtig decken aktive, professionelle Experten nur noch einen Bruchteil der paläarktischen Käferfauna ab. Ohne Amateure und pensionierte Exprofessionelle wäre die Verwirklichung eines Kataloges wie des unseren undenkbar.

Man spricht viel über Biodiversität und „hot-spots“, aber die artenreichen Gruppen, die solche „spots“ charakterisieren, kann kaum jemand bestimmen. Die groß angelegten Biodiversitäts-Programme, ausgeführt in den so stark gefährdeten Tropen und Subtropen, erbringen Material, das nur ganz oberflächlich ausgewertet wird. Die Ökologie scheint auf Artbestimmungen immer weniger Wert zu legen, für zahlreiche Studien greift man zu „Modell-Gruppen“, die Bestimmungsprobleme beiseite schieben, und man arbeitet mit dem Begriff „Morphospezies“, der doch in der Realität nur ein Name (ein Buchstabe oder eine Ziffer) ist, unter dem eine unbekannte Zahl artverschiedener Organismen verborgen sein kann.

\section{Was ist eigentlich Taxonomie und was Systematik?}

Das Leben existiert in Form diskreter Einheiten. Diese sind formalisiert und werden als Taxa bezeichnet. Als grundlegendes Taxon ist der Begriff „Art“ allgemein anerkannt (die Art kann als die „kleinste identifizierbare Einheit“, kladistisch (evolutionär) oder biologisch definiert sein). Verschiedene verwandte Arten, die eine vermutliche oder reale monophyletische Gruppe bilden, sind in Gattungen und dann weiter in höheren 
Taxa geordnet zusammengefasst. Dieses Vorgehen entspricht der Kategorisierung von Gegenständen und Beobachtungen, die, unter anderem, dem Menschen das abstrakte Denken und eine Orientierung in seiner Umgebung ermöglichen. Das wusste schon der alte Aristoteles, entgeht aber manchen modernen Biologen. Das strukturierte Erfassen des Lebens in einem System ist eine Vorrausetzung dafür, Informationen über jede einzelne Art oder andere Taxa zu gewinnen und mitteilen zu können. Begriffe wie „Faunen“, „Ökosysteme“ und „Biodiversität“ wären sinnlos ohne Bezug auf ein solches System.

Eine zweite Vorraussetzung ist ein universell anerkanntes Benennungsvorgehen, die Nomenklatur. Es wäre sinnlos, Taxa zu definieren und zu klassifizieren, ohne sie gleichzeitig mit Hilfe universell geltender Namen zu kodieren.

Die Wissenschaft, die sich mit der Entdeckung und Definition von Arten und anderen Taxa beschäftigt, ist die Taxonomie. Die eng mit ihr zusammenhängende Systematik ist mit der Klassifikation der Taxa in einem natürlichen System verbunden; beide zusammen suchen sie nach Merkmalen, die den Taxa eigen sind, sowie nach solchen, die für die Rekonstruktion der phylogenetischen Beziehungen relevant sind. Taxonomie und Systematik sind für Informationen aufgeschlossen, die aus verschiedensten Bereichen stammen, unter anderem aus der Embryologie, Physiologie, Ethologie, Ökologie, der Verbreitung, sowie auch der molekularen Biologie. Darüber hinaus stehen taxonomische Hypothesen (Validität der Taxa, Verwendbarkeit der Merkmale) immer wieder unter Verifikationsdruck.

Trotzdem wird die Taxonomie eher als ein aussterbender Zweig der Vergangenheit, als eine verstaubte museale Disziplin oder als eine lediglich beschreibende, essentialistische und nicht wissenschaftliche Tätigkeit angesehen.

Man fragt sich, ob sie in der heutigen Welt überhaupt noch eine Berechtigung hat. Nötige Kredite werden jedenfalls gekürzt. In den Universitäten wird sie, zusammen mit der Systematik, allmählich zu minimalen Systemüberblicken reduziert. Die Folgen spiegeln sich in den vorher erwähnten Problemen wider: die theoretischen Grundlagen der Taxonomie und Systematik befinden sich außerhalb der allgemeinen Ausbildungsprogramme.

Die Verwaltungen der Museen neigen immer mehr dazu, der pädagogischen Tätigkeit und der Erfassung der Sammlungsbelege Priorität zu geben. Hingegen wird die Forschung im Bereich der Taxonomie oft nur geduldet. Die größeren Museen besitzen Sammlungen, die in vielen Bereichen eine verlässliche Erfassung der phänotypischen Plastizität ermöglichen. Eine andere wichtige Vorraussetzung für taxonomische Studien in Museen sind die Fachbibliotheken. Trotzdem werden vielfach auch dort molekular-technische Methoden, die Vergleichsammlungen und umfangreiche Bibliotheken nicht brauchen, auf Kosten der alpha-Taxonomie unterstützt.

Es stellt sich nun die Frage, woher kommt es, dass in den höheren Entscheidungssphären Taxonomie und Systematik so negativ beurteilt oder als uninteressant abgestuft werden? Die Ursachen dafür sind offenbar komplex. Folgende sollen hier diskutiert werden:

1. Die Beschreibungen der Taxa bestehen aus langweiligen Texten und einfachen Zeichungen oder Photographien. Dem Unorientierten bleibt verborgen, dass der Taxonom einzelne Merkmale prüft und versucht, jene herauszuarbeiten, die für die untersuchten Taxa wirklich charakteristisch sind. Lebewesen sind variabel, oft sexuell polymorph und können sich unter dem Druck der außerhalb von ihnen herrschenden Umstände verwandeln. Deswegen macht es oft Schwierigkeiten, manche Taxa sicher voneinander zu unterscheiden, obwohl es technisch leicht ist, Millionen Individuen eindeutig zu definieren. 
Die Bestimmungstabellen, die jedem mit minimalen taxonomischen Kenntnissen Ausgerüsteten die Identifizierung einer Art ermöglichen, sind Resultate langer Untersuchung, welche nicht selten an vielen tausenden Belegen zahlreicher Populationen verifiziert worden sind. Dass, beispielsweise, hinter dem mit trivialen Worten ausgedrückten Bestimmungsmerkmal eines Schädlings (das 5. Antennenglied ist länger als das 6.) eine lange Forschungsgeschichte liegt, entgeht dem unorientierten Benutzer.

Es ist paradox, dass Bestimmungen, die Derivate der Taxonomie sind, für Taxonomie gehalten werden. Es ist fast so, als wenn man das Ausschreiben eines Rezepts für medizinische Forschung hielte!

2) Formelle taxonomische Entscheidungen sind partiell willkürlich (auch wenn sie auf gut fundierten Hypothesen beruhen), was den Eindruck einer „Nicht-Wissenschaft“ erwecken mag. Das Willkürliche ist unabhängig von der angewandten Methodik und eigentlich am deutlichsten dort, wo molekulare Daten phenetisch ausgewertet werden (bedenken wir die vorgeschlagene prozentuelle „Unterschiedsgrenze“, die Arten voneinander trennen soll: nach der $5 \%$ Grenze gehören wir und die Schimpansen zu ein und derselben Art). Merkwürdigerweise spricht man auch über eine $2 \%$ oder $3 \%$ „Unterschiedsgrenze“, nicht aber über 2,5\%.

3) Man erwartet ein abgeschlossenes Resultat. Seit Linnaeus' 10. Edition der Systema Naturae hat man nur einen kleinen Teil der rezenten Arten weltweit erfasst. Daher die folgende arithmetische Extrapolation: „250 Jahre brauchte man für etwa 400.000 Käferarten; 1250 Jahre wird man für 2 Millionen Arten benötigen“. Die Arithmetiker haben aber etwas übersehen: seit Linnaeus' Zeiten versuchen Taxonomen die bereits erkannten Taxa mit neu entdeckten Merkmalen und neuen Bewertungen genauer zu definieren. Der Großteil der Arbeit der meisten Taxonomen in den letzten zweieinhalb Jahrhunderten ist von solchen „Revisionen“ ausgefüllt.

Es ist auch erstaunlich, dass von der Taxonomie, und nur von der Taxonomie, eine Endgültigkeit verlangt wird - solche Ansprüche sind normalerweise doch jeder Wissenschaft fremd.

4) Wissenschaft ist auch eine kompetitive Angelegenheit und die Bedeutung wissenschaftlicher Arbeit wird immer mehr nach einem „citation index“ und „impact factor" beurteilt. Der „citation index“ wird aus den Veröffentlichungen der vorherigen 2 Jahre ermittelt. In dieser kurzen Spanne werden aber nur 1-2 \% der taxonomischen Publikationen überhaupt zitiert. Im Durchschnitt sind taxonomische Referenzen nämlich über 60 Jahre, für kurze Studien über 40 Jahre alt (KöHLER 1988, Krell 2002) und nur 1,5\% decken die Spanne des „Science Citation Index“. Kurze Studien mit einer „allgemein“ orientierten Diskussion im Bereich starker Forscherpopulationen verkaufen sich weit besser in gut quotierten Periodika als umfassende Monographien vernachlässigter Taxa. Zu umfangreiche, als Bücher publizierte Werke, werden vom technokratisch kalkulierten „impact factor“ nicht berücksichtigt und können, wie Publikationen in nicht indexierten Periodika, sogar karriereschädlich sein. Dass taxonomische Studien, im Unterschied zu der Mehrzahl anderer biologischer Arbeiten, auch noch nach langen Zeitspannen verwendet und zitiert werden, interessiert die entscheidenden Stellen wenig. Darüber hinaus favorisiert der „impact factor“ das Modische und der „citation index“ sogar die Publikation von durchaus unüberzeugenden Resultaten, wie z. B. der „Fall“ Ecdysozoa (siehe Pilato et al. 2005) zeigt.

5) Es wird einfach nicht zur Kenntnis genommen, dass man in allen biologischen Studien konkrete Taxa untersucht ohne Bezug zur Taxonomie, die doch die Voraussetzung für die Verständigung bildet. 
6) Die nomenklatorischen Regeln müssen formell und universal gültig sein. Sie berücksichtigen nicht die qualitativen Aspekte einer Studie. Daher hinterlassen auch die schlampigsten taxonomischen Publikationen eine Spur, die, wenn es um neu eingeführte taxonomische Namen geht, von anderen Taxonomen berücksichtigt werden muss. In diesem Punkt ist die Taxonomie gegenüber allen anderen biologischen Bereichen benachteiligt.

7) Es wird auch die Frage gestellt, ob es überhaupt Sinn hat zu wissen, welche Käfer (oder Wanzen) in einem Wald oder inmitten einer Wüste leben? Vielleicht hat es tatsächlich wenig Sinn. Analoge Frage können wir jedenfalls auch in anderen Bereichen stellen. Ist es nur eine Vermutung, dass das Leben auf der Erde uns näher steht als z. B. die Existenz von Millionen Lichtjahren entfernten Sternen, deren Studium unproportional höher als die Taxonomie dotiert ist?

\section{Auswege}

1. Phylokodisten kamen mit der Idee, die nicht monophyletischen Taxa, die hierarchische (strukturierte) Klassifikation und das Typuskonzept zu verwerfen (De QueIroz \& GaUtier 1990). Sie haben sich für „revolutionär“ erklärt und die bisherige Taxonomie mit der Nomenklatur als kreationistisch und essentialistisch abgewertet. Sie wollen ein ganz neues Benennungsverfahren einführen: die Namen müssen auf phylogenetisch belegten „clades“ beruhen und einmal akzeptiert unverändert bleiben. Dass phylogenetische Analysen zahlreiche evolutive Szenarios bieten und dass Änderungen in der Auswahl der Merkmale und Taxa auch zu Änderungen der phylogenetischen Hypothesen führen können (siehe auch JENNER 2004), wird kaum zu Kenntnis genommen. Ähnlich werden auch die Konfusionen zwischen Homoplasien und Apomorphien weitgehend ignoriert. Die Versuche, die Sache in die Praxis umzusetzen, sollen erst ab 2006 laufen. Das neue Verfahren könnte den Computer-Fans die Möglichkeit bieten, die bisherigen Tiernamen zu ersetzen, ohne eine kritische (und mühsame) Untersuchung der relevanten Merkmale. Doch, wie PicketT (2005) zeigte, wird die mit viel Energie und Aggressivität propagierte Idee mit hohe Wahrscheinlichkeit bald in Vergessenheit geraten.

2. Eine Reduktion der langen Beschreibungen soll, nach ERwIN \& JoHnson (2000), ökonomischer mit der Zeit der Taxonomen und ihrem Papier verfahren. Überraschenderweise wird dabei aber das Folgende nicht zu Kenntnis genommen: die eigene Beschreibungszeit ist für einen erfahrenen Experten irrelevant, und die eventuelle Einsparung von einer halben oder sogar einer ganzen Stunde spielt kaum eine Rolle im Vergleich zu der Zeit, die für das Anfertigen von Präparaten, Abbildungen, Messungen, für Vergleiche und für das Erstellen von Bestimmungstabellen verwendet werden muss. Eine zusätzliche Beschreibung im Internet mag Geld, nicht aber die Zeit der Taxonomen sparen; dazu zeigt die Erfahrung, dass „,vorläufig“ und kurz definierte Taxa selten durch eine detaillierte Arbeit desselben Autors ersetzt werden.

3. Die taxonomische Information soll im Internet (wie Intergrated Taxonomic Information System, Dokumente in der Form PDF, Automontage) publiziert sein (siehe Godfray 2002). Sie wird dadurch allgemein und leicht zugänglich. So praktisch und so billig es sein mag, im Internet zu publizieren, eine Qualitätskontrolle bleibt dabei auf der Strecke. Außerdem kann es dadurch noch schwieriger werden, parallel publizierte, widersprüchliche Konzepte zu behandeln. Hervorragende Abbildungen von Belegen, die repräsentativ für Taxa sind 
(z. B. Holotypen) können mit Automontage und Internet ein breites Publikum erreichen. Für die praktische Bestimmungsarbeit funktioniert dies dort, wo taxonomische Probleme schon ausgeräumt sind und nicht gleichzeitig viele sehr ähnliche Arten sympatrisch vorkommen. Sie haben die Vor- und Nachteile aller Ikonographien, sind aber im Vergleich zu den letzteren viel billiger und qualitativ überlegen. Nicht oder beschränkt verwendbar sind solche Abbildungen dort, wo eine grundlegende taxonomische Arbeit fehlt, wenn nötiges, sicher bestimmtes Material nicht zugänglich oder in nicht geeignetem Zustand ist, und wenn die Variabilität der Merkmale nicht gezeigt werden kann.

Die Internet- und die Automontage-Techniken können den Fortschritt in taxonomischen Studien erheblich beschleunigen, nicht aber die Taxonomie selbst ersetzen.

4. Molekulare Merkmale charakterisieren Taxa. Einmal entnommen und in Datenbasen (GenBank) via Internet zugänglich, würden molekulare Merkmale eine sichere Bestimmung der Organismen ermöglichen. Experten-Taxonomen würden dann überflüssig sein. Die DNA mitochondrial oxydase subunit 1 ist in Form von „Barcodes“ als Träger solcher Information vorgeschlagen worden. Diese Idee wurde unter anderem in Nature, Science, The Economist und Der Spiegel für breiteste Kreise propagiert. Die mit dieser Technik verbundenen Probleme sollen hier nicht diskutiert werden (doch siehe SEBERG et al. 2003, Will \& Rubinoff 2004), abgesehen von der Bemerkung, dass die geographische Variabilität der als Modell definierten Sequenzen weitgehend ignoriert wird. Dabei ist es bekannt, dass Arten oft polymorphe Haplotypen mit Divergenzen enthalten, die älter als die Spezies-Divergenzen sind (Sperling 2003). Abgesehen von technischen Problemen soll man jedenfalls folgendes bedenken:

* DNA-Taxonomie muss auf Belegen, die a) nicht zu alt (vielleicht nicht viel mehr als 5 Jahre) und auf geeignete Weise abgetötet sind und, in den meisten Fällen, b) von Taxonomen bestimmt sind, beruhen. Dies ist auch von den Gründern der „Barcodes“ anerkannt, im Widerspruch zu dem gleichzeitig postulierten „raison d'être" der Barcodes, das Ersetzen der Experten-Taxonomen (siehe TAuTz et al. 2003). Ein großer Teil der Käfer wie auch der übrigen Arthropoden ist einfach nicht von Experten betreut, und es ist auch unmöglich, in absehbarer Zeit frische Belege aller bekannten Arten einzusammeln.

* Auch hier gibt es eine Konfusion der Begriffe: die Taxonomie wird mit ihrer Bestimmungsfunktion identifiziert.

* Die Definition von Taxa beruht auf Fragmenten eines oder von wenigen Genen, die auf der Morphologie basierenden Taxonomie arbeitet mit Merkmals-Komplexen, die auf zahlreichen Genen fußen.

* Ein auf Barcodes beruhendes Identifikationsverfahren führt zu zwei parallelen „Welten“: einem, das aus „barcoded“ Arten besteht, einem zweiten, dem „klassischen". Das erste wird schätzungsweise kaum die Hälfte der rezenten und nicht die ausgestorbene Käferarten enthalten können.

* Menschliches Interesse, Lernen und Können werden grob unterschätzt. Man braucht wenig Aufwand, um die Ordnungen und sehr viele Familien (und Gattungen) der Insekten auseinander zu halten und anzusprechen zu können. Diese Information ist fast umsonst und kann auch in angewandten Bereichen nützlich sein. Sie stellt oft den ersten Schritt dar, der zum Studium der Natur führt. Aus rein menschlicher Sicht kommt die Reduktion der Organismen zu den benannten molekularen Fragmenten einer Reduktion von Kunstwerken auf einige von ihnen entnommene Moleküle gleich. 
Vielleicht ist es noch interessant zu bemerken, dass, wie bei vielen anderen einfachen Rezepten, die es den Menschen versprechen, Probleme abzuschaffen, auch das auf Barcodes basierende Rezept auf Glauben beruht.

\section{Wie soll es weitergehen?}

Wir haben einen Fragebogen ausgearbeitet, um klarere Vorstellungen über Taxonomen und Taxonomie zu gewinnen (Löbl \& Leschen 2005). Dieser wurde an 154 Mitarbeiter des "Catalogue of Palaearctic Coleoptera" verschickt, 104 davon haben geantwortet. Nicht ausgewertet sind Daten von den beiden Autoren des Fragebogens.

Die meisten Mitarbeiter stammen aus Mitteleuropa, es gibt aber auch zahlreiche aus Skandinavien, Russland, Italien, Spanien, Japan, Nordamerika sowie auch aus Australien und Neuseeland. Es scheint symptomatisch für die gegenwärtige Lage der Taxonomie zu sein, dass wir nur 4 aus England gewinnen konnten, davon sind 3 pensioniert, einer, aktiv, kam ursprünglich aus Deutschland. Aus Frankreich stammen nur 3 Mitarbeiter, keiner davon ist ein professioneller Biologe.

Die Auswertung ergab das Folgende:

- $60 \%$ haben ein Universitätsstudium mit dem Doktorat abgeschlossen

- $70 \%$ sind aktiv

- Das Durchschnittsalter beträgt 50 Jahre

- 39 \% arbeiten in Museen, $30 \%$ privat, $26 \%$ in Universitäten

- $88 \%$ arbeiten ohne besondere Kredite (abgesehen vom Gehalt bei den Professionellen) und ohne Assistenz

- im Durchschnitt hat jeder 99 Arten beschrieben

- $91 \%$ arbeiten zu Hause (inklusive der professionellen Taxonomen)

- $28 \%$ beteiligt sich an molekularen Programmen

- $78 \%$ halten DNA-Studien potenziell nützlich

- $35 \%$ halten barcoding für nutzlos, $23 \%$ für nützlich, $39 \%$ sind ohne Meinung

- $50 \%$ halten Phylocode für nutzlos, $8 \%$ für nützlich, und $40 \%$ sind ohne Meinung

Unsere Daten sind statistisch nicht überzeugend. Sie geben aber einen partiellen Einblick und lassen Fragen unbeantwortet. Wir brauchen neue Analysen von anderen und größeren Populationen. Aus den Kommentaren, die uns mitgeteilt wurden, lässt sich immerhin eine fast einstimmige Meinung herauslesen, was die Bewertung molekularer Methoden in der Taxonomie und Phylogenie betrifft:

- molekulare Daten haben einen schwachen Aussagewert, wenn sie von anderen Daten, besonders morphologischen, abgekoppelt werden,

- taxonomische und phylogenetische Studien, die molekulare Daten berühren, werden von Leuten, die über Kredite entscheiden, a priori besser bewertet als Studien, die andere Techniken verwenden, 
- das gegenwärtige System funktioniert, weil die molekular-technisch ausgebildeten Biologen entweder selber gleichzeitig Taxonomen sind, oder von Taxonomen die notwendige Information bekommen, oder Bereiche untersuchen, in denen keine Bestimmungsproblematik existiert.

Wer die gegenwärtigen Tendenzen extrapoliert, kommt zu dem Ergebnis: an den Universitäten vieler Länder werden kaum mehr Taxonomie-Experten ausgebildet. Die Museen, die als die letzten Bastionen der professionellen Taxonomie eine einmalige Funktion haben, werden also in der Zukunft jene Biologen anstellen müssen, die am Arbeitsmarkt verfügbar sind, nämlich molekular-technologische Experten. Unsere Kenntnisse und Erkenntnisse über die Lebensformen (und ihre Rolle in der Natur) werden dann unter Umständen ganz auf den Amateuren lasten - die von der professionellen Biologie gezwungener Weise abgekoppelt werden.

In Nordamerika und im Fernen Osten wird die Bedeutung der Taxonomie nicht unterschätzt. Dort erfahren schon Studenten, dass molekulare Merkmale nicht mehr und nicht weniger als eine Informationsquelle sind, die zusammen mit anderen Merkmalskomplexen auszuwerten ist. Vielleicht kommt also eine Wende von Westen? Oder aus dem Osten und in der Zukunft werden auch die taxonomischen Arbeiten nach Europa importiert.

Effizienter natürlich wäre eine von a priori befreite Wertung aller biologischen Disziplinen. Neue Technologien hatten immer eine große Anziehungskraft. Sie sollten aber nicht die Arbeit an der Klärung von Grundfragen schwächen, wie es im Falle der Taxonomie und Systematik gegenwärtig in Europa geschieht (z. B. Disney 1999, Wheeler 2004). Es ist zu hoffen, dass dies auch von den verantwortlichen Stellen zur Kenntnis genommen wird.

\section{Literatur}

Dejean, P. F. M. A. 1821: Catalogue de la collection de Coléoptères de M. le Baron Dejean. - Paris: Crevot, viii $+136+2$ (errata) $S$.

De Queiroz, K. \& Gautier, J. 1990: Phylogeny as a central principle in taxonomy: Phylogenetic definition of taxon name. - Systematic Zoology 39: 307-322.

Disney, R. H. L. 1999: Insect biodiversity and the demise of alpha taxonomy. - Antenna 23: 84-88.

ERwin, T. L. \& Johnson, P. J. 2000: Naming species, a new paradigm for crisis management in taxonomy: rapid journal validation of scientific names enhanced with more complete descriptions on the internet. - The Coleopterists Bulletin 54: 269-278.

FARRIS, J. S. 1974: Formal definitions of paraphyly and polyphyly. - Systematic Zoology 23: 548-554.

Godfray, C. J. 2002: Challenges for taxonomy. - Nature 417: 17-19.

Hennig, W. 1966: Phylogenetic systematics. - University of Illinois Press, Urbana: 263 S.

JeNNER, R. A. 2004: The scientific status of metazoan cladfistics: why currant research practise must change. - Zoologica Scripta 33: 293-310.

KöHLER, F. 1988: Amateurwissenschaft: Entwicklung, Beschreibung und wissenschaftliche Analyse am Beispiel der Koleopterologie. - Diploma Thesis: Sociologie. Universität Köln: 195 S.

KreLL, F.-H. 2002: Why impact factors don't work for taxonomy. - Nature 415: 957.

LinNaEus, C. 1767: Systema Naturae per regna tria naturae, secundum classes, ordines, genera, species, cum characteribus, differentiis, synonymis, locis. Tom I. Pars II. Editio Duodecima Reformata. - Holmiae: Impensis Direct. Laur. Salvii, 533-1327 + [37] S.

Löвl, I. \& Leschen, R. A. B. 2005: Demography of coleopterists and their thoughts on DNA barcoding and the Phylocode, with commentary. - The Coleopterists Bulletin 59: 284-292. 
Löbl, I. \& Smetana, A. 2003: Catalogue of Palaearctic Coleoptera. Vol. 1. - Stenstrup, Apollo Books: 819 S.

Löbl, I. \& Smetana, A. 2004: Catalogue of Palaearctic Coleoptera. Vol. 2. - Stenstrup, Apollo Books: $942 \mathrm{~S}$.

Pickett, M. K. 2005: The new and improved Phylocode, now with types, ranks, and even polyphyly: a conference report from the First International Phylogenetic Nomenclature Meeting. - Cladistics 21: 79-82.

Pilato G.; Binda, M. G.; Biondi O.; D’Urso, V.; Lisi, O.; Marletta, A.; Maugeri, S.; Nobile, V.; Rappazzo G.; Sabella, G.; Sammartano, F.; Turrisi, G. \& Viglisnisi F. 2005: The clade Ecdysozoa, perplexities and questions. - Zoologischer Anzeiger 244: 43-50.

Seberg, O.; Humpries, C. J.; Knapp, S.; Stevenson, D. W.; Petersen, G.; Scharff, N. \& Andersen, N. M. 2003: Shortcuts in systematics? A commentary on DNA-based taxonomy. - Trends in Ecology and Evolution 18: 63-66.

Sperling, F. 2003: DNA Barcoding: Deus ex Machina. - Newsletter of the Biological survey of Canada (Terrestrial Arthropods) 22: 50-53.

Tautz, D.; Arctander, P.; Minelli, A.; Thomas, R. H. \& Vogler, A. F. 2003: A plea for DNA taxonomy. - Trends in Ecology and Evolution 18: 70-74.

Wheeler, Q. D. 1995: The "Old Systematics": classification and phylogeny [pp. 31-62]. - In: Biology, Phylogeny, and Classification of Coleoptera. Papers Celebrating the $80^{\text {th }}$ Birthday of Roy A. Crowson. Vol. 1 (J. Pakaluk and S. A. Slipinski, eds). Muzeum i Instytut Zoologii PAN, Warszawa.

Wheeler, Q. D. 2004: Taxonomic triage and the poverty of phylogeny. - Philosophical Transactions of the Royal Society of London B 359: 571-583.

Wiley, E. O. 1981: Phylogenetics. The Theory and Practice of Phylogenetic Systematics. - John Wiley \& Sons, New York, Chichester, Brisbane, Toronto, Singaporex: 439 S.

Will, K. W. \& Rubinoff, D. 2004: Myth of the molecule: DNA barcodes for species cannot replace morphology for identification and classification. - Cladistics 20: 47-55.

\section{Anschrift des Verfassers:}

Dr. IVAN LÖBL

Muséum d'histoire naturelle

Case postale 6434

$\mathrm{CH}$ - 1211 Genève 6

Schweiz

e-mail: ivan.lobl@bluewin.ch

\section{Subject editor:}

B. KLAusNitzer 